\title{
ASYMPTOTIC EXPANSIONS FOR ANISOTROPIC HEAT KERNELS
}

\author{
LIVIU I. IGNAT AND ENRIQUE ZUAZUA
}

\begin{abstract}
We obtain the asymptotic expansion of the solutions of some anisotropic heat equations when the initial data belong to polynomially weighted $L^{p}$-spaces. We mainly address two model examples. In the first one, the diffusivity is of order two in some variables but higher in the other ones. In the second one we consider the heat equation on the Heisenberg group.
\end{abstract}

\section{INTRODUCTION}

This paper is devoted to the asymptotic behavior as time tends to infinity for the Cauchy problem associated with some anisotropic heat equations, with initial data in polynomially weighted $L^{p}$ spaces.

There are a number of results on asymptotic behavior of the classical isotropic heat equation on the whole space:

$$
\begin{cases}u_{t}(z, t)=\Delta u(z, t) & z \in \mathbb{R}^{N}, t>0, \\ u(z, 0)=f(z), & , z \in \mathbb{R}^{N}\end{cases}
$$

The key to obtain a complete asymptotic expansion of solutions as $t \rightarrow \infty$ is a decomposition of the initial data on the basis constituted by Dirac's delta and its derivatives, proved in [3] and that we recall for the sake of completeness. The asymptotic expansion reads as follows:

Let $G(\cdot, t)$ be the heat kernel. For any $1 \leq p \leq N /(N-1)$ and $k \geq 0$ an integer the solution of problem (1.1) satisfies:

$$
\left\|u(\cdot, t)-\sum_{|\alpha| \leq k} \frac{(-1)^{|\alpha|}}{\alpha !}\left(\int_{\mathbb{R}^{N}} f(z) z^{\alpha} d z\right) D^{\alpha} G(\cdot, t)\right\|_{L^{p}\left(\mathbb{R}^{N}\right)} \lesssim t^{-\frac{k+1}{2}}\left\||z|^{k+1} f\right\|_{L^{p}\left(\mathbb{R}^{N}\right)}
$$

for any initial data $f \in L^{1}\left(\mathbb{R}^{N}, 1+|z|^{k}\right)$ such that $|z|^{k+1} f \in L^{p}\left(\mathbb{R}^{N}\right)$.

We refer to [3] for a proof and various extensions.

According to this expansion the asymptotic behavior of solutions of the heat equation can be described in terms of the Gaussian heat kernel and its space derivatives, using the moments of the initial data as coefficients.

This decomposition is of isotropic nature; all space variables are treated equally, with the same homogeneity. This fact does not permit dealing with anisotropic heat kernels that would require different decomposition results, weighting the various euclidean variables 
differently, or even with isotropic heat kernels, as the one associated with the heat equation (1.1), but with initial data in anisotropically weighted spaces.

In this paper we present new decomposition results for the initial data that lead both to new results for the heat equation (1.1) and that, simultaneously, allow handling a number of anisotropic heat kernels.

We focus mainly on two model examples to illustrate the key ideas and the phenomena that emerge due to anisotropy.

The first one is the following diffusion equation, of mixed order, namely order two in some of the space variables, and order four in the other ones:

$$
\begin{cases}u_{t}(z, t)=\Delta_{x}^{2} u(z, t)+\Delta_{y} u(z, t), & z=(x, y) \in \mathbb{R}^{m} \times \mathbb{R}^{n}, t>0, \\ u(z, 0)=f(z), & z \in \mathbb{R}^{m+n} .\end{cases}
$$

The solution $u$ can be written in convolution form as $u(t)=G_{t} * f$ where $G_{t}$ is the fundamental solution

$$
G_{t}(x, y)=t^{-\frac{m}{2}-\frac{n}{4}} G_{1}\left(\frac{x}{t^{1 / 4}}, \frac{y}{t^{1 / 2}}\right)
$$

where the similarity profile $G_{1}$ is a smooth function.

Based on the decomposition obtained in [3] we get a new one (see Lemma 3.1 below) allowing us to prove the following asymptotic expansion.

Theorem 1.1. Let $k$ be a positive odd integer. For any $f \in L^{1}\left(\mathbb{R}^{m+n}, 1+|x|^{k+1}+|y|^{\frac{k+1}{2}}\right)$ the solution $u$ of system (1.2) satisfies

$$
\begin{gathered}
\left\|u(\cdot, t)-\sum_{|\beta|+2|\gamma| \leq k} \frac{(-1)^{|(\beta, \gamma)|}}{\beta ! \gamma !}\left(\int_{\mathbb{R}^{N}} f(x, y) x^{\beta} y^{\gamma} d x d y\right) D_{x y}^{\beta \gamma} G_{t}\right\|_{L^{1}\left(\mathbb{R}^{N}\right)} \\
\lesssim t^{-\frac{k+1}{4}}\left\|\left(1+|x|^{k+1}+|y|^{\frac{k+1}{2}}\right) f\right\|_{L^{1}\left(\mathbb{R}^{N}\right)}
\end{gathered}
$$

Observe that the assumptions on the initial data weigh differently the various euclidean variables, taking into account the inhomogeneity of the diffusion operator. The resulting decomposition is in agreement with the different weight that the euclidean variables have in the fundamental solution, so that the overall contribution of its different derivatives is balanced. For simplicity we only have stated the result in the $L^{1}\left(\mathbb{R}^{m+n}\right)$-norms but similar results can be stated in $L^{p}\left(\mathbb{R}^{m+n}\right)$ spaces under some restrictions on the exponent $p$. The decomposition in [3] would be insufficient to obtain such results since it is of isotropic nature, thus weighting equally all space variables, which is not sufficient to deal with the anisotropy of the heat kernel under consideration. The method presented in this paper allows treating separately the euclidean variables with different homogeneity.

As we shall see, the decomposition used to obtain this result can also be applied in the case of the classical isotropic heat equation, leading to new results on its asymptotic decomposition as $t \rightarrow \infty$. 
The second problem we address is the heat equation on the Heisenberg group:

$$
\begin{cases}u_{t}(z, \theta, t)=\Delta_{\mathbb{H}^{n}} u(z, \theta, t) & (z, \theta) \in \mathbb{R}^{2 n} \times \mathbb{R}, t>0, \\ u(z, \theta, 0)=f(z, \theta), & ,(z, \theta) \in \mathbb{R}^{2 n} \times \mathbb{R} .\end{cases}
$$

Let us recall that, as for the classical heat equation, the heat semi-group on $\mathbb{H}^{n}$ is given by the convolution between the initial datum and the fundamental solution, a function in the Schwartz class (see [4]).

Theorem 1.2. (4]) There exists a function $H \in \mathcal{S}\left(\mathbb{R}^{2 n+1}\right)$ such that, $u$, the solution of the heat equation on the Heisenberg group (1.4) is given by

$$
u(\cdot, t)=f * H_{t},
$$

where $*$ is the convolution on the Heisenberg group while $H_{t}$ is defined by

$$
H_{t}(z, \theta)=\frac{1}{t^{n+1}} H\left(\frac{z}{\sqrt{t}}, \frac{\theta}{t}\right) .
$$

Moreover, the function $H$ can be computed explicitly (cf. [4])

$$
H((z, \theta))=\frac{1}{(4 \pi)^{n+1}} \int_{\mathbb{R}}\left(\frac{2 \sigma}{\sinh (2 \sigma)}\right)^{n} \exp \left(\frac{i \sigma \theta}{2}-\frac{|z|^{2}}{2} \frac{\sigma}{\tanh 2 \sigma}\right) d \sigma .
$$

In this case we obtain the following asymptotic expansion.

Theorem 1.3. For any $f \in L^{1}\left(\mathbb{R}^{2 n+1}, 1+|z|^{2}+|\theta|\right)$ the solution $u$ of problem (1.4) with initial data $f$ satisfies the following

$$
\begin{aligned}
\left\|u(\cdot, t)-\left(\int_{\mathbb{H}^{n}} f\right) H_{t}+\sum_{j=1}^{2 n}\left(\int_{\mathbb{H}^{n}} z_{j} f(z, \theta)\right) Z_{j} H_{t}\right\|_{L^{1}\left(\mathbb{R}^{2 n+1}\right)} & \\
& \lesssim t^{-1}\left\|\left(1+|z|^{2}+|\theta|\right) f\right\|_{L^{1}\left(\mathbb{R}^{2 n+1}\right)}, \quad t>0
\end{aligned}
$$

where $Z_{j}, j=1, \ldots, 2 n$ are the first $2 n$ vector fields of the Lie algebra on $\mathbb{H}^{n}$.

The results presented here can be extended to more general situations, for example to the heat equation on homogeneous Carnot groups. We refer to [6] where this issue is addressed, although the results in that paper do not take into account the different homogeneity in what concerns the needed assumptions on the initial data. Note also the existing results for heat equations in heterogeneous media. For instance in [5] by combining the results in [3] and Bloch-wave expansions a complete asymptotic expansion is given for the heat equation in periodic media. We also refer to [2] for similar expansions in the context of Vlasov - Poisson - Fokker - Planck equations.

The paper is organized as follows. In Section 2 we consider the classical isotropic heat equation to illustrate that the use of the new decomposition results we present here leads to new asymptotic expansions as $t \rightarrow \infty$ that can not be derived out of [3]. In Section 3 we consider the anisotropic model (1.2), proving Theorem 1.1 and we compare it with the asymptotic decomposition results one could get out of the isotropic decomposition results in [3]. In the last section we present some classical facts about the Heisenberg group and give the proof of the corresponding asymptotic expansion stated in Theorem 1.3 . 


\section{Decomposition Lemmas}

In this section we obtain some variants of the results in [3] on the decomposition of functions defined in the euclidean space in the basis of Dirac's deltas and its derivatives. We shall derive some applications to the asymptotic behavior of the isotropic heat equation for initial data in anisotropically weighted spaces.

In what follows we denote by $\mathcal{D}\left(\mathbb{R}^{N}\right)$ the space of $C^{\infty}$ and compactly supported functions and by $\mathcal{D}^{\prime}\left(\mathbb{R}^{N}\right)$ its dual.

We first recall the following decomposition [3].

Lemma 2.1. ([3]) Let $k$ be a positive integer. For any $f \in \mathcal{D}\left(\mathbb{R}^{N}\right)$ it holds

$$
f=\sum_{|\alpha| \leq k} \frac{(-1)^{|\alpha|}}{\alpha !}\left(\int_{\mathbb{R}^{N}} f(x) x^{\alpha} d x\right) D^{\alpha} \delta_{0}+\sum_{|\alpha|=k+1} D^{\alpha} F_{\alpha},
$$

where the functions $F_{\alpha}$, defined for $|\alpha|=k+1$, are given by

$$
F_{\alpha}(x)=(k+1) \frac{(-1)^{k+1}}{\alpha !} \int_{0}^{1}(1-t)^{k}\left(\frac{x}{t}\right)^{\alpha} f\left(\frac{x}{t}\right) \frac{d t}{t^{N}} .
$$

Moreover, for any $p \in\left[1, \frac{N}{N-1}\right)$ the following holds

$$
\left\|F_{\alpha}\right\|_{L^{p}\left(\mathbb{R}^{N}\right)} \leq\left\||x|^{k+1} f\right\|_{L^{p}\left(\mathbb{R}^{N}\right)} .
$$

Remark 1. By density, the above result holds for all $f \in L^{1}\left(\mathbb{R}^{N}, 1+|x|^{k}\right)$ with $|x|^{k+1} f \in$ $L^{p}\left(\mathbb{R}^{N}\right)$.

The above result is a consequence of Taylor's formula with integral remainder, applied to any test function $\varphi$ :

$$
\varphi(z)=\sum_{|\alpha| \leq k}\left(D^{\alpha} \varphi\right)(0) \frac{z^{\alpha}}{\alpha !}+(k+1) \sum_{|\alpha|=k+1} \frac{z^{\alpha}}{\alpha !} \int_{0}^{1}(1-t)^{k}\left(D^{\alpha} \varphi\right)(t z) d t, z \in \mathbb{R}^{N} .
$$

We now derive some anisotropic variants. For, given $z \in \mathbb{R}^{N}, N \geq 2$ we decompose it as $z=(x, y) \in \mathbb{R}^{m} \times \mathbb{R}^{n}$ with $m+n=N$. We then use a splitting argument and apply first Taylor's expansion in the $y$ variable with $x$ fixed and then perform Taylor's expansion in $x$.

Lemma 2.2. For any $\varphi \in \mathcal{D}\left(\mathbb{R}^{N}\right)$ the following holds

$$
\begin{gathered}
\varphi(z)=\sum_{|\alpha| \leq k}\left(D^{\alpha} \varphi\right)(0) \frac{z^{\alpha}}{\alpha !}+\sum_{|\gamma| \leq k}\left[(k+1-|\gamma|) \sum_{|\beta|=k+1-\gamma} \frac{x^{\beta}}{\beta !} \int_{0}^{1}(1-t)^{k+|\gamma|}\left(D_{x}^{\beta} D_{y}^{\gamma} \varphi\right)(t x, 0) d t\right] \frac{y^{\gamma}}{\gamma !} \\
+(k+1) \sum_{|\gamma|=k+1} \frac{y^{\gamma}}{\gamma !} \int_{0}^{1}(1-t)^{k}\left(D_{y}^{\gamma} \varphi\right)(x, t y) d t, \quad z=(x, y) \in \mathbb{R}^{N} .
\end{gathered}
$$


Proof. Using Taylor's expansion in $y$ with $x$ fixed we get

$$
\varphi(z)=\varphi(x, y)=\sum_{|\gamma| \leq k}\left(D_{y}^{\gamma} \varphi\right)(x, 0) \frac{y^{\gamma}}{\gamma !}+(k+1) \sum_{|\gamma|=k+1} \frac{y^{\gamma}}{\gamma !} \int_{0}^{1}(1-t)^{k}\left(D_{y}^{\gamma} \varphi\right)(x, t y) d t .
$$

Now, applying Taylor's expansion with respect to $x$ we get:

$$
\begin{aligned}
\varphi(z)= & \sum_{|\gamma| \leq k}\left[\sum_{|\beta| \leq k-|\gamma|}\left(D_{x}^{\beta} D_{y}^{\gamma} \varphi\right)(0,0) \frac{x^{\beta}}{\beta !} \frac{y^{\gamma}}{\gamma !}\right] \\
& +\sum_{|\gamma| \leq k}\left[(k+1-|\gamma|) \sum_{|\beta|=k+1-\gamma} \frac{x^{\beta}}{\beta !} \int_{0}^{1}(1-t)^{k+|\gamma|}\left(D_{x}^{\beta} D_{y}^{\gamma} \varphi\right)(t x, 0) d t\right] \frac{y^{\gamma}}{\gamma !} \\
& +(k+1) \sum_{|\gamma|=k+1} \frac{y^{\gamma}}{\gamma !} \int_{0}^{1}(1-t)^{k}\left(D_{y}^{\gamma} \varphi\right)(x, t y) d t .
\end{aligned}
$$

Writing $\alpha=(\beta, \gamma)$ we obtain the desired identity.

We now introduce the space $L^{p}\left(\mathbb{R}^{m},|x|^{|\beta|} ; L^{1}\left(\mathbb{R}^{n},|y|^{|\gamma|}\right)\right)$, constituted by the functions $f$ such that $|x|^{|\beta|}\left\||y|^{|\gamma|} f(x, \cdot)\right\|_{L^{1}\left(\mathbb{R}^{n}\right)}$ belongs to $L^{p}\left(\mathbb{R}^{m}\right)$, i.e.:

$$
\|f\|_{L^{p}\left(\mathbb{R}^{m},|x|^{|\beta|} ; L^{1}\left(\mathbb{R}^{n},|y| \gamma \mid\right)\right)}^{p}=\int_{\mathbb{R}^{m}}|x|^{p|\beta|}\|f(x, \cdot)\|_{L^{1}\left(\mathbb{R}^{n} ;|y|^{|\gamma|}\right)}^{p} d x<\infty .
$$

Following the same ideas of treating differently the different variables $x$ and $y$ we get the following result.

Lemma 2.3. For any $f \in \mathcal{D}\left(\mathbb{R}^{m+n}\right)$ it holds

$$
\begin{aligned}
f(x, y)= & \sum_{|(\beta, \gamma)| \leq k} \frac{(-1)^{|(\beta, \gamma)|}}{\beta ! \gamma !}\left(\int_{\mathbb{R}^{N}} f(x, y) x^{\beta} y^{\gamma} d x d y\right) D_{x y}^{\beta \gamma} \delta_{0} \\
& +\sum_{|\gamma| \leq k|\beta|=k+1-|\gamma|} \sum_{|\gamma|} \frac{(-1)^{|\gamma|}}{\gamma !} D_{x}^{\beta}[\mathcal{R} f]_{\beta \gamma}(x) D_{y}^{\gamma} \delta_{0}(y) \\
& +\sum_{|\gamma|=k+1} D_{y}^{\gamma} F_{\gamma}(x, y), \quad(x, y) \in \mathbb{R}^{m} \times \mathbb{R}^{n}
\end{aligned}
$$

where the remainder terms are defined as follows:

$$
[\mathcal{R} f]_{\beta \gamma}(x)=\frac{(-1)^{|\beta|}|\beta|}{\beta !} \int_{0}^{1}(1-t)^{|\beta|-1}\left(\frac{x}{t}\right)^{\beta} \int_{\mathbb{R}^{n}} f\left(\frac{x}{t}, y\right) y^{\gamma} d y \frac{d t}{t^{m}}
$$

for $|\gamma| \leq k,|\beta|+|\gamma|=k+1$, and

$$
F_{\gamma}(x, y)=(k+1) \frac{(-1)^{k+1}}{\gamma !} \int_{0}^{1}(1-t)^{k}\left(\frac{y}{t}\right)^{\gamma} f\left(x, \frac{y}{t}\right) \frac{d t}{t^{n}}
$$

for $|\gamma|=k+1$. 
Moreover, for $1 \leq p<\frac{n}{n-1}$ it holds

$$
\left\|F_{\gamma}\right\|_{L^{p}\left(\mathbb{R}^{N}\right)} \leq\left\||y|^{k+1} f\right\|_{L^{p}\left(\mathbb{R}^{N}\right)}
$$

and for $1 \leq p<\frac{m}{m-1}$ it holds

$$
\left\|[\mathcal{R} f]_{\beta \gamma}\right\|_{L^{p}\left(\mathbb{R}^{m}\right)} \leq\|f\|_{L^{p}\left(\mathbb{R}^{m},|x|^{|\beta|} ; L^{1}\left(\mathbb{R}^{n},|y|^{|\gamma|}\right)\right)} .
$$

Remark 2. The main term is the same as in Lemma [2.1, since

$$
\sum_{|(\beta, \gamma)| \leq k} \frac{(-1)^{|(\beta, \gamma)|}}{\beta ! \gamma !}\left(\int_{\mathbb{R}^{N}} f(x, y) x^{\beta} y^{\gamma} d x d y\right) D_{x y}^{\beta \gamma} \delta_{0}=\sum_{|\alpha| \leq k} \frac{(-1)^{|\alpha|}}{\alpha !}\left(\int_{\mathbb{R}^{N}} f(z) z^{\alpha} d z\right) D^{\alpha} \delta_{0} .
$$

But estimating the remainder terms $F_{\gamma}$ and $[M f]_{\beta \gamma}$ different anisotropic spaces from those of Lemma 2.1 are needed.

By density, the decomposition holds for all $f \in L^{1}\left(\mathbb{R}^{N}, 1+|\cdot|^{k}\right)$ that belongs to

$$
\mathcal{X}^{p}=\bigcap_{|\gamma| \leq k} L^{p}\left(\mathbb{R}^{m},|x|^{k+1-|\gamma|} ; L^{1}\left(\mathbb{R}^{n},|y|^{|\gamma|}\right)\right) \bigcap L^{p}\left(\mathbb{R}^{m} ; L^{p}\left(\mathbb{R}^{n},|y|^{k+1}\right)\right) .
$$

We also define the natural norm on $\mathcal{X}^{p}$

$$
\|f\|_{\mathcal{X}^{p}}:=\sum_{0 \leq|\gamma| \leq k}\|f\|_{L_{x}^{p}\left(\mathbb{R}^{m},|x|^{k+1-|\gamma|} ; L_{y}^{1}\left(\mathbb{R}^{n},|y|^{|\gamma|}\right)\right)}+\left\||y|^{k+1} f\right\|_{L^{p}\left(\mathbb{R}^{N}\right)} .
$$

When $p=1$ the space $\mathcal{X}^{p}$ is exactly the one needed in Lemma 2.1, i.e. $L^{1}\left(\mathbb{R}^{m+n},|(x, y)|^{k+1}\right)$.

Proof of Lemma 2.3. We write $\mathbb{R}^{N}$ as $\mathbb{R}^{m} \times \mathbb{R}^{n}$. We first fix $x \in \mathbb{R}^{m}$ and apply Lemma 2.1 to the function $y \rightarrow f(x, y)$. Then

$$
f(x, y)=\sum_{|\gamma| \leq k} \frac{(-1)^{|\gamma|}}{\gamma !}\left(\int_{\mathbb{R}^{n}} f(x, y) y^{\gamma} d y\right) D_{y}^{\gamma} \delta_{0}(y)+\sum_{|\gamma|=k+1} D_{y}^{\gamma} F_{\gamma}(x, y)
$$

where

$$
F_{\gamma}(x, y)=(k+1) \frac{(-1)^{k+1}}{\gamma !} \int_{0}^{1}(1-t)^{k}\left(\frac{y}{t}\right)^{\gamma} f\left(x, \frac{y}{t}\right) \frac{d t}{t^{n}} .
$$

A similar argument as in [3] shows that for any $x \in \mathbb{R}^{m}$ and $p \in\left[1, \frac{n}{n-1}\right)$ we have

$$
\left\|F_{\gamma}(x, \cdot)\right\|_{L^{p}\left(\mathbb{R}^{n}\right)} \leq\left\||\cdot|^{k+1} f(x, \cdot)\right\|_{L^{p}\left(\mathbb{R}^{n}\right)} .
$$

Taking the $L^{p}\left(\mathbb{R}^{m}\right)$-norm in the $x$-variable we obtain estimate (2.11).

We denote by $[M f]_{\gamma}(x)$ the $y$-moment of order $\gamma$ of $f(x, \cdot)$ in $\mathbb{R}^{n}$ :

$$
[M f]_{\gamma}(x)=\int_{\mathbb{R}^{n}} f(x, y) y^{\gamma} d y
$$

We apply Lemma 2.1 to the function $x \rightarrow[M f]_{\gamma}(x)$ and obtain

$$
[M f]_{\gamma}(x)=\sum_{|\beta| \leq k-|\gamma|} \frac{(-1)^{|\beta|}}{\beta !}\left(\int_{\mathbb{R}^{m}}[M f]_{\gamma}(x) x^{\beta} d x\right) D_{x}^{\beta} \delta_{0}(x)+\sum_{|\beta|=k+1-|\gamma|} D_{x}^{\beta}[\mathcal{R} f]_{\beta \gamma}(x)
$$


where $[\mathcal{R} f]_{\beta \gamma}$ is given by

$$
[\mathcal{R} f]_{\beta \gamma}(x)=\frac{(-1)^{|\beta|}|\beta|}{\beta !} \int_{0}^{1}(1-t)^{|\beta|-1}\left(\frac{x}{t}\right)^{\beta}[M f]_{\gamma}\left(\frac{x}{t}\right) \frac{d t}{t^{m}} .
$$

Writing explicitly the function $[M f]_{\gamma}$, we have

$$
[M f]_{\gamma}(x)=\sum_{|\beta| \leq k-|\gamma|} \frac{(-1)^{|\beta|}}{\beta !}\left(\int_{\mathbb{R}^{N}} f(x, y) x^{\beta} y^{\gamma} d x d y\right) D_{x}^{\beta} \delta_{0}(x)+\sum_{|\beta|=k+1-|\gamma|} D_{x}^{\beta}[\mathcal{R} f]_{\beta \gamma}(x)
$$

where $[\mathcal{R} f]_{\beta \gamma}$ are given by

$$
[\mathcal{R} f]_{\beta \gamma}(x)=\frac{(-1)^{|\beta|}|\beta|}{\beta !} \int_{0}^{1}(1-t)^{|\beta|-1}\left(\frac{x}{t}\right)^{\beta} \int_{\mathbb{R}^{n}} f\left(\frac{x}{t}, y\right) y^{\gamma} d y \frac{d t}{t^{m}} .
$$

Replacing $[M f]_{\gamma}$ in (2.13) with identity (2.17) we obtain

$$
\begin{aligned}
f= & \sum_{|\gamma| \leq k} \sum_{|\beta| \leq k-|\gamma|} \frac{(-1)^{|\gamma|+|\beta|}}{\gamma ! \beta !}\left(\int_{\mathbb{R}^{N}} f(x, y) x^{\beta} y^{\gamma} d x d y\right) D_{x}^{\beta} \delta_{0}(x) D_{y}^{\gamma} \delta_{0}(y) \\
& +\sum_{|\gamma| \leq k} \sum_{|\beta|=k+1-|\gamma|} \frac{(-1)^{|\gamma|}}{\gamma !} D_{x}^{\beta}[\mathcal{R} f]_{\beta \gamma}(x) D_{y}^{\gamma} \delta_{0}(y)+\sum_{|\gamma|=k+1} D_{y}^{\gamma} F_{\gamma}(x, y) \\
= & \sum_{|(\beta, \gamma)| \leq k} \frac{(-1)^{|(\beta, \gamma)|}}{\beta ! \gamma !}\left(\int_{\mathbb{R}^{N}} f(x, y) x^{\beta} y^{\gamma} d x d y\right) D_{x y}^{\beta \gamma} \delta_{0} \\
& +\sum_{|\gamma| \leq k} \sum_{|\beta|=k+1-|\gamma|} \frac{(-1)^{|\gamma|}}{\gamma !} D_{x}^{\beta}[\mathcal{R} f]_{\beta \gamma}(x) D_{y}^{\gamma} \delta_{0}(y)+\sum_{|\gamma|=k+1} D_{y}^{\gamma} F_{\gamma}(x, y) .
\end{aligned}
$$

Proceeding as in [3] and using Minkowski's inequality, for any $1 \leq p<\frac{m}{m-1}$ we get estimate (2.12) on the remainder $[\mathcal{R} f]_{\beta \gamma}$

$$
\left\|[\mathcal{R} f]_{\beta \gamma}\right\|_{L^{p}\left(\mathbb{R}^{m}\right)} \leq\left\||x|^{\beta} \int_{\mathbb{R}^{n}} f(x, y) y^{\gamma} d y \mid\right\|_{L^{p}\left(\mathbb{R}^{m}\right)} \leq\|f\|_{L^{p}\left(\mathbb{R}^{m},|x| \beta \mid ; L^{1}\left(\mathbb{R}^{n},|y||\gamma|\right)\right)} .
$$

The proof is now finished.

We now apply the new decomposition obtained in Lemma 2.3 to the heat equation (1.1).

Theorem 2.1. Assume that $N=m+n$. For any $1 \leq p<\min \left\{\frac{m}{m-1}, \frac{n}{n-1}\right\}$ and $k \geq 0$ integer, the solution of problem (1.1) satisfies:

$$
\left\|u(\cdot, t)-\sum_{|\alpha| \leq k} \frac{(-1)^{|\alpha|}}{\alpha !}\left(\int_{\mathbb{R}^{N}} f(z) z^{\alpha} d z\right) D^{\alpha} G(\cdot, t)\right\|_{L^{p}\left(\mathbb{R}^{N}\right)} \lesssim t^{-\frac{k+1}{2}}\|f\|_{\mathcal{X}^{p}}
$$

for any initial data $f \in L^{1}\left(\mathbb{R}^{N}, 1+|z|^{k}\right) \cap \mathcal{X}^{p}$. 
Remark 3. In the case $p=1$ the above estimate is the same as the one obtained in [3] by applying Lemma 2.1.

The range of exponents $p$ for which the above Theorem applies is larger than that in [3] but the space $\mathcal{X}^{p}$ where the initial data is taken is more complex and of anisotropic nature.

Proof. We now apply Lemma 2.3 to the initial data $f$. Then

$$
\begin{aligned}
& \left\|G(t) * f-\sum_{|\alpha| \leq k} \frac{(-1)^{|\alpha|}}{\alpha !}\left(\int_{\mathbb{R}^{N}} f(x) x^{\alpha} d x\right) D^{\alpha} G(t)\right\|_{L^{p}\left(\mathbb{R}^{N}\right)} \\
& \leq \sum_{0 \leq|\gamma| \leq k} \sum_{|\beta|=k+1-|\gamma|}\left\|G(t) *\left(D_{x}^{\beta}[\mathcal{R} f]_{\beta \gamma}(x) D_{y}^{\gamma} \delta_{0}(y)\right)\right\|_{L^{p}\left(\mathbb{R}^{N}\right)}+\sum_{|\gamma|=k+1}\left\|G(t) * D_{y}^{\gamma} F_{\gamma}(x, y)\right\|_{L^{p}\left(\mathbb{R}^{N}\right)} \\
& \leq \sum_{0 \leq|\gamma| \leq k} \sum_{|\beta|=k+1-|\gamma|}\left\|D_{x}^{\beta} D_{y}^{\gamma} G(t, \cdot, y) *_{x}[\mathcal{R} f]_{\beta \gamma}(\cdot)\right\|_{L^{p}\left(\mathbb{R}^{N}\right)}+\sum_{|\gamma|=k+1}\left\|D_{y}^{\gamma} G(t) * F_{\gamma}(x, y)\right\|_{L^{p}\left(\mathbb{R}^{N}\right)} \\
& \leq \sum_{0 \leq|\gamma| \leq k|\beta|=k+1-|\gamma|} \sum_{\|}\left\|D_{x}^{\beta} D_{y}^{\gamma} G(t)\right\|_{L_{x}^{1}}\left\|[\mathcal{R} f]_{\beta \gamma}\right\|_{L_{x}^{p}}\left\|_{L_{y}^{p}}+|t|^{-\frac{k+1}{2}}\right\| F_{\gamma} \|_{L^{p}\left(\mathbb{R}^{N}\right)}
\end{aligned}
$$

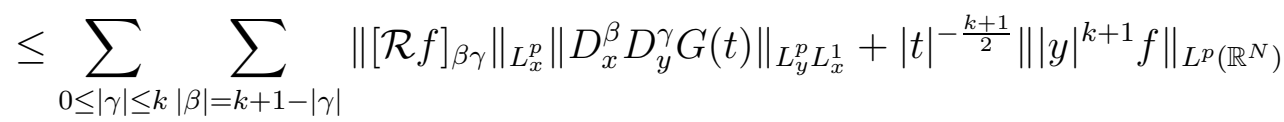

$$
\begin{aligned}
& \leq \sum_{0 \leq|\gamma| \leq k} \sum_{|\beta|=k+1-|\gamma|}|t|^{-\frac{|\beta|+|\gamma|}{2}-\frac{n}{2}\left(1-\frac{1}{p}\right)}\|f\|_{L^{p}\left(\mathbb{R}^{m},|x||\beta| ; L^{1}\left(\mathbb{R}^{n},|y||\gamma|\right)\right)}+|t|^{-\frac{k+1}{2}}\left\||y|^{k+1} f\right\|_{L^{p}\left(\mathbb{R}^{N}\right)} \\
& \leq t^{-\frac{k+1}{2}}\|f\|_{\mathcal{X}^{p}}
\end{aligned}
$$

The proof of Theorem 2.1 is now finished.

\section{An Anisotropic heat EQUATIOn of MiXed ORDER}

This section is devoted to the analysis of the following system

$$
\begin{cases}u_{t}(z, t)=\Delta_{x}^{2} u(z, t)+\Delta_{y} u(z, t), & z=(x, y) \in \mathbb{R}^{m} \times \mathbb{R}^{n}, t>0, \\ u(z, 0)=f(z), & z \in \mathbb{R}^{m+n} .\end{cases}
$$

The solution $u$ of the above system is given by $u(t)=G_{t} * f$ where $G_{t}$ is the fundamental solution given by

$$
G_{t}(x, y)=t^{-\frac{m}{4}-\frac{n}{2}} G_{1}\left(\frac{x}{t^{1 / 4}}, \frac{y}{t^{1 / 2}}\right),
$$

and the self-similar profile $G_{1}$ is so that its Fourier transform is such that

$$
\hat{G}_{1}(\xi, \eta)=e^{-|\xi|^{4}-|\eta|^{2}} .
$$

We now apply Lemma 2.1 to the initial data $f$. For any $1 \leq p<N /(N-1), N=m+n$, we obtain that $u$, solution of (3.18), satisfies

$$
\left\|u(\cdot, t)-\sum_{|\alpha| \leq k} \frac{(-1)^{|\alpha|}}{\alpha !}\left(\int_{\mathbb{R}^{N}} f(z) z^{\alpha} d z\right) D^{\alpha} G_{t}(\cdot, t)\right\|_{L^{p}\left(\mathbb{R}^{N}\right)} \lesssim\left\|\left.z\right|^{k+1} f\right\|_{L^{p}\left(\mathbb{R}^{N}\right)} \sum_{|\alpha|=k+1}\left\|D^{\alpha} G_{t}\right\|_{L^{1}\left(\mathbb{R}^{N}\right)},
$$


or, writing each index $\alpha$ of length $N=n+m$ as $\alpha=(\beta, \gamma) \in \mathbb{Z}^{m} \times \mathbb{Z}^{n}$,

$$
\begin{array}{r}
\left\|u(\cdot, t)-\sum_{|(\beta, \gamma)| \leq k} \frac{(-1)^{|(\beta, \gamma)|}}{\beta ! \gamma !}\left(\int_{\mathbb{R}^{N}} f(x, y) x^{\beta} y^{\gamma} d x d y\right) D_{x y}^{\beta \gamma} G_{t}\right\|_{L^{p}\left(\mathbb{R}^{N}\right)} \\
\quad \lesssim\left\|\left.z\right|^{k+1} f\right\|_{L^{p}\left(\mathbb{R}^{N}\right)} \sum_{|\beta|+|\gamma|=k+1}\left\|D_{x}^{\beta} D_{y}^{\gamma} G_{t}\right\|_{L^{1}\left(\mathbb{R}^{N}\right)} .
\end{array}
$$

Remark that, due to the anisotropy of the fundamental solution, the derivatives in the $x$-variable decay differently from those in the $y$-variable:

$$
\left\|D_{x}^{\beta} D_{y}^{\gamma} G_{t}\right\|_{L^{q}\left(\mathbb{R}^{N}\right)} \simeq t^{-\frac{|\beta|}{4}-\frac{|\gamma|}{2}-\frac{N}{2}\left(1-\frac{1}{q}\right)}, \quad q \in[1, \infty] .
$$

As a consequence, for large $t$ we have

$$
\begin{aligned}
\| u(\cdot, t)- & \sum_{|(\beta, \gamma)| \leq k} \frac{(-1)^{|(\beta, \gamma)|}}{\beta ! \gamma !}\left(\int_{\mathbb{R}^{N}} f(x, y) x^{\beta} y^{\gamma} d x d y\right) D_{x y}^{\beta \gamma} G_{t} \|_{L^{p}\left(\mathbb{R}^{N}\right)} \\
& \lesssim\left\|\left.z\right|^{k+1} f\right\|_{L^{p}\left(\mathbb{R}^{N}\right)} \sum_{|\beta|+|\gamma|=k+1} t^{-\frac{|\beta|}{4}-\frac{|\gamma|}{2}} \lesssim t^{-\frac{k+1}{4}}\left\||z|^{k+1} f\right\|_{L^{p}\left(\mathbb{R}^{N}\right)}
\end{aligned}
$$

Note however that in the left hand side of the above inequality there are terms that decay faster than the remainder $t^{-\frac{k+1}{4}}$. Accordingly it is natural to split the terms in the left hand side in two parts: those that decay faster and, respectively, slower than $t^{-\frac{k+1}{4}}$.

Define

$$
\Lambda(p, k)=\left\{(a, b) \in \mathbb{Z}^{2}: a, b \geq 0, k+1-2 N(1-1 / p) \leq a+2 b, a+b \leq k\right\} .
$$

It follows that, as long as $f$ belongs to some weighted spaces, the solution $u$ of system (3.18) satisfies for large time $t$ the following estimate

$$
\begin{aligned}
& \left\|u(\cdot, t)-\sum_{|\beta|+2|\gamma|<k+1-2 N(1-1 / p)} \frac{(-1)^{|(\beta, \gamma)|}}{\beta ! \gamma !}\left(\int_{\mathbb{R}^{N}} f(x, y) x^{\beta} y^{\gamma} d x d y\right) D_{x y}^{\beta \gamma} G_{t}\right\|_{L^{p}\left(\mathbb{R}^{N}\right)} \\
& \lesssim t^{-\frac{k+1}{4}}\left\||z|^{k+1} f\right\|_{L^{p}\left(\mathbb{R}^{N}\right)}+\sum_{(\beta, \gamma) \in \Lambda(p, k)} t^{-\frac{|\beta|}{4}-\frac{|\gamma|}{2}-\frac{N}{2}\left(1-\frac{1}{p}\right)}\|f\|_{L^{1}\left(\mathbb{R}^{N},|x||\beta||| y|\gamma|\right)} \\
& \lesssim t^{-\frac{k+1}{4}}\left(\left\||z|^{k+1} f\right\|_{L^{p}\left(\mathbb{R}^{N}\right)}+\sum_{(\beta, \gamma) \in \Lambda(p, k)}\|f\|_{L^{1}\left(\mathbb{R}^{N},|x||\beta||y||\gamma|\right)}\right) .
\end{aligned}
$$

We now state rigorously the result to which this analysis leads. For the sake of simplicity we state it for $p=1$ only. 
Theorem 3.1. For any $f \in L^{1}\left(\mathbb{R}^{n+m}, 1+|x|^{k+1}+|y|^{k+1}\right)$ the following holds for large time $t$ :

$$
\begin{aligned}
\| u(\cdot, t)- & \sum_{|\beta|+2|\gamma| \leq k} \frac{(-1)^{|(\beta, \gamma)|}}{\beta ! \gamma !}\left(\int_{\mathbb{R}^{N}} f(x, y) x^{\beta} y^{\gamma} d x d y\right) D_{x y}^{\beta \gamma} G_{t} \|_{L^{1}\left(\mathbb{R}^{N}\right)} \\
& \lesssim t^{-\frac{k+1}{4}}\left\|\left(1+|x|^{k+1}+|y|^{k+1}\right) f\right\|_{L^{1}\left(\mathbb{R}^{N}\right)}
\end{aligned}
$$

Proof. We apply estimate (3.20) to obtain the following:

$$
\begin{aligned}
\| u(\cdot, t)- & \sum_{|\beta|+2|\gamma| \leq k} \frac{(-1)^{|(\beta, \gamma)|}}{\beta ! \gamma !}\left(\int_{\mathbb{R}^{N}} f(x, y) x^{\beta} y^{\gamma} d x d y\right) D_{x y}^{\beta \gamma} G_{t} \|_{L^{1}\left(\mathbb{R}^{N}\right)} \\
& \lesssim t^{-\frac{k+1}{4}}\left(\left\||z|^{k+1} f\right\|_{L^{1}\left(\mathbb{R}^{N}\right)}+\sum_{(\beta, \gamma) \in \Lambda(p, k)}\|f\|_{L^{1}\left(\mathbb{R}^{N},\left.|x|^{|\beta|}|y|\right|^{|\gamma|}\right)}\right) \\
& \lesssim t^{-\frac{k+1}{4}}\left(\left\|\left(|x|^{k+1}+|y|^{k+1}\right) f\right\|_{L^{1}\left(\mathbb{R}^{N}\right)}+\left\|\left(|x|^{k}+|y|^{k}\right) f\right\|_{L^{1}\left(\mathbb{R}^{N}\right)}\right) .
\end{aligned}
$$

Since $f$ belongs to $L^{1}\left(\mathbb{R}^{N}\right)$ we obtain the desired result.

Observe that, in view of (3.19), we need twice more derivatives in $x$ than in $y$ to obtain the same decay of the kernel $G_{t}$. We now give a new decomposition that, when applied to the anisotropic equation (3.18), distinguishes the two variables $x$ and $y$.

We consider the case when $k$ is an odd integer, $k+1=2 m+2$. Since we have seen that $\left\|D_{x}^{\alpha} G_{t}\right\|_{L^{p}\left(\mathbb{R}^{N}\right)} \simeq\left\|D_{y}^{\beta} G_{t}\right\|_{L^{p}\left(\mathbb{R}^{N}\right)},|\alpha|=2|\beta|=2 m$, we will take into account that, for the decay rate of the kernel, one derivative in $y$ has the same effect as two derivatives in $x$.

Lemma 3.1. Let $k$ be a positive odd integer. For any $f \in \mathcal{D}\left(\mathbb{R}^{m+n}\right)$ it holds

$$
\begin{aligned}
f(x, y)= & \sum_{|\beta|+2|\gamma| \leq k} \frac{(-1)^{|(\beta, \gamma)|}}{\beta ! \gamma !}\left(\int_{\mathbb{R}^{N}} f(x, y) x^{\beta} y^{\gamma} d x d y\right) D_{x y}^{\beta \gamma} \delta_{0} \\
& +\sum_{|\gamma| \leq(k-1) / 2} \sum_{|\beta|+2|\gamma|=k+1} \frac{(-1)^{|\gamma|}}{\gamma !} D_{x}^{\beta}[\mathcal{R} f]_{\beta \gamma}(x) D_{y}^{\gamma} \delta_{0}(y)+\sum_{|\gamma|=(k+1) / 2} D_{y}^{\gamma} F_{\gamma}(x, y)
\end{aligned}
$$

where the remainder terms are defined as follows:

$$
F_{\gamma}(x, y)=\frac{k+1}{2} \frac{(-1)^{\frac{k+1}{2}}}{\gamma !} \int_{0}^{1}(1-t)^{\frac{k-1}{2}}\left(\frac{y}{t}\right)^{\gamma} f\left(x, \frac{y}{t}\right) \frac{d t}{t^{n}},
$$

for $|\gamma|=\frac{k+1}{2}$, and

$$
[\mathcal{R} f]_{\beta \gamma}(x)=\frac{(-1)^{|\beta|}|\beta|}{\beta !} \int_{0}^{1}(1-t)^{|\beta|-1}\left(\frac{x}{t}\right)^{\beta} \int_{\mathbb{R}^{n}} f\left(\frac{x}{t}, y\right) y^{\gamma} d y \frac{d t}{t^{m}}
$$

defined for $|\gamma| \leq(k-1) / 2,|\beta|+2|\gamma|=k+1$. 
Moreover, for $1 \leq p<\frac{n}{n-1}$, it holds

$$
\left\|F_{\gamma}\right\|_{L^{p}\left(\mathbb{R}^{N}\right)} \leq\left\||y|^{\frac{k+1}{2}} f(x, \cdot)\right\|_{L^{p}\left(\mathbb{R}^{N}\right)}
$$

and, for $1 \leq p<\frac{m}{m-1}$

$$
\left\|[\mathcal{R} f]_{\beta \gamma}\right\|_{L^{p}\left(\mathbb{R}^{m}\right)} \leq\|f\|_{L^{p}\left(\mathbb{R}^{m},|x||\beta| ; L^{1}\left(\mathbb{R}^{n},|y||\gamma|\right)\right)} .
$$

Remark 4. By density the results hold true for a larger class of functions $f$.

Proof of Lemma 3.1. Set $k+1=2 l+2$. Fix $x \in \mathbb{R}^{m}$ and apply Lemma 2.1 to the function $y \rightarrow f(x, y)$. Then

$$
f(x, y)=\sum_{|\gamma| \leq l} \frac{(-1)^{|\gamma|}}{\gamma !}\left(\int_{\mathbb{R}^{n}} f(x, y) y^{\gamma} d y\right) D_{y}^{\gamma} \delta_{0}(y)+\sum_{|\gamma|=l+1} D_{y}^{\gamma} F_{\gamma}(x, y)
$$

where

$$
F_{\gamma}(x, y)=(l+1) \frac{(-1)^{l+1}}{\gamma !} \int_{0}^{1}(1-t)^{l}\left(\frac{y}{t}\right)^{\gamma} f\left(x, \frac{y}{t}\right) \frac{d t}{t^{n}},|\gamma|=l+1 .
$$

Observe that for any $x \in \mathbb{R}^{m}$ and $p \in\left[1, \frac{n}{n-1}\right)$ we have

$$
\left\|F_{\gamma}(x, \cdot)\right\|_{L^{p}\left(\mathbb{R}^{n}\right)} \leq\left\||y|^{l+1} f(x, \cdot)\right\|_{L^{p}\left(\mathbb{R}^{n}\right)} .
$$

Taking the $L^{p}\left(\mathbb{R}^{m}\right)$-norm in the $x$ variable we obtain estimate (3.22).

Recall the definition of $[M f]_{\gamma}$ given in (2.16). We apply Lemma 2.1 to the function $x \rightarrow[M f]_{\gamma}(x)$, with a remainder of order $k+1-|\gamma|$ and obtain that

$$
[M f]_{\gamma}(x)=\sum_{|\beta| \leq k-2|\gamma|} \frac{(-1)^{|\beta|}}{\beta !}\left(\int_{\mathbb{R}^{m}}[M f]_{\gamma}(x) x^{\beta} d x\right) D_{x}^{\beta} \delta_{0}(x)+\sum_{|\beta|=k+1-2|\gamma|} D_{x}^{\beta}[\mathcal{R} f]_{\beta \gamma}(x),
$$

where

$$
[\mathcal{R} f]_{\beta \gamma}(x)=\frac{(-1)^{|\beta|}|\beta|}{\beta !} \int_{0}^{1}(1-t)^{|\beta|-1}\left(\frac{x}{t}\right)^{\beta}[M f]_{\gamma}\left(\frac{x}{t}\right) \frac{d t}{t^{m}} .
$$


Using decomposition (3.27) in (3.24) we obtain that $f$ can be written as

$$
\begin{aligned}
f(x, y)= & \sum_{|\gamma| \leq m} \frac{(-1)^{|\gamma|}}{\gamma !}\left(\sum_{|\beta| \leq k-2|\gamma|} \frac{(-1)^{|\beta|}}{\beta !}\left(\int_{\mathbb{R}^{N}} f(x, y) x^{\beta} y^{\gamma} d x d y\right) D_{x}^{\beta} \delta_{0}(x)+\right. \\
& \left.+\sum_{|\beta|=k+1-2|\gamma|} D_{x}^{\beta}[\mathcal{R} f]_{\beta \gamma}(x)\right) D_{y}^{\gamma} \delta_{0}(y) \\
& +\sum_{|\gamma|=m+1} D_{y}^{\gamma} F_{\gamma}(x, y) \\
= & \sum_{|\gamma| \leq m|\beta| \leq k-2|\gamma|} \frac{(-1)^{|\gamma|}}{\gamma !} \frac{(-1)^{|\beta|}}{\beta !}\left(\int_{\mathbb{R}^{N}} f(x, y) x^{\beta} y^{\gamma} d x d y\right) D_{x}^{\beta} \delta_{0}(x) D_{y}^{\gamma} \delta_{0}(y) \\
& +\sum_{|\gamma| \leq m|\beta|=k+1-2|\gamma|} \frac{(-1)^{|\gamma|}}{\gamma !} D_{x}^{\beta}[\mathcal{R} f]_{\beta \gamma}(x) D_{y}^{\gamma} \delta_{0}(y)+\sum_{|\gamma|=m+1} D_{y}^{\gamma} F_{\gamma}(x, y) \\
= & \sum_{|\beta|+2|\gamma| \leq 2 m+1} \frac{(-1)^{|(\beta, \gamma)|}}{\beta ! \gamma !}\left(\int_{\mathbb{R}^{N}} f(x, y) x^{\beta} y^{\gamma} d x d y\right) D_{x y}^{\beta \gamma} \delta_{0} \\
& +\sum_{|\gamma| \leq m|\beta|=2 m+2-|\gamma|} \sum_{\frac{(-1)^{|\gamma|}}{\gamma !} D_{x}^{\beta}[\mathcal{R} f]_{\beta \gamma}(x) D_{y}^{\gamma} \delta_{0}(y)+\sum_{|\gamma|=m+1} D_{y}^{\gamma} F_{\gamma}(x, y) .}
\end{aligned}
$$

Estimate (3.23) on the remainder $[\mathcal{R} f]_{\beta \gamma}$ is obtained as follows: for any $1 \leq p<\frac{m}{m-1}$ we have

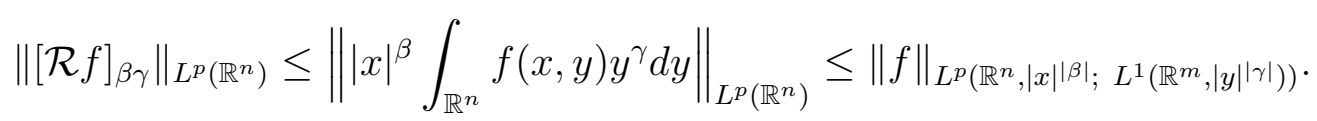

The proof is now complete.

We now apply this new decomposition to obtain an asymptotic expansion for the solutions of (3.18), and prove Theorem 1.1. 
Proof of Theorem 1.1. We now apply Lemma 3.1 to our initial data $f \in L^{1}\left(\mathbb{R}^{N}, 1+|x|^{k+1}+\right.$ $\left.|y|^{\frac{k+1}{2}}\right)$. Then

$$
\begin{aligned}
& \left\|G_{t} * f-\sum_{|\beta|+2|\gamma| \leq k} \frac{(-1)^{\mid(\beta, \gamma)}}{\beta ! \gamma !}\left(\int_{\mathbb{R}^{N}} f(x) x^{\beta} y^{\gamma} d x d y\right) D_{x y}^{\beta \gamma} G_{t}\right\|_{L^{1}\left(\mathbb{R}^{N}\right)} \\
& \leq \sum_{|\gamma| \leq(k-1) / 2} \sum_{|\beta|+2|\gamma|=k+1}\left\|G_{t} *\left(D_{x}^{\beta}[\mathcal{R} f]_{\beta \gamma}(x) D_{y}^{\gamma} \delta_{0}(y)\right)\right\|_{L^{1}\left(\mathbb{R}^{N}\right)} \\
& +\sum_{|\gamma|=(k+1) / 2}\left\|G_{t} * D_{y}^{\gamma} F_{\gamma}(x, y)\right\|_{L^{1}\left(\mathbb{R}^{N}\right)} \\
& \leq \sum_{|\gamma| \leq(k-1) / 2} \sum_{|\beta|+2|\gamma|=k+1}\left\|D_{x}^{\beta} D_{y}^{\gamma} G_{t}(\cdot, y) *_{x}[\mathcal{R} f]_{\beta \gamma}(\cdot)\right\|_{L^{1}\left(\mathbb{R}^{N}\right)} \\
& +\sum_{|\gamma|=(k+1) / 2}\left\|D_{y}^{\gamma} G_{t} * F_{\gamma}(x, y)\right\|_{L^{1}\left(\mathbb{R}^{N}\right)} \\
& \leq \sum_{0 \leq|\gamma| \leq k|\beta|=k+1-|\gamma|} \sum_{\|}\left\|D_{x}^{\beta} D_{y}^{\gamma} G_{t}\right\|_{L_{x}^{1}\left(\mathbb{R}^{m}\right)}\left\|[\mathcal{R} f]_{\beta \gamma}\right\|_{L_{x}^{1}\left(\mathbb{R}^{m}\right)}\left\|_{L_{y}^{1}\left(\mathbb{R}^{n}\right)}+|t|^{-\frac{k+1}{4}} \sum_{|\gamma|=(k+1) / 2}\right\| F_{\gamma} \|_{L^{1}\left(\mathbb{R}^{N}\right)} \\
& \leq \sum_{|\gamma| \leq(k-1) / 2} \sum_{|\beta|+2|\gamma|=k+1}\left\|[\mathcal{R} f]_{\beta \gamma}\right\|_{L_{x}^{1}}\left\|D_{x}^{\beta} D_{y}^{\gamma} G_{t}\right\|_{L^{1}\left(\mathbb{R}^{N}\right)}+|t|^{-\frac{k+1}{4}}\left\||y|^{\frac{k+1}{2}} f\right\|_{L^{1}\left(\mathbb{R}^{N}\right)} \\
& \leq \sum_{|\gamma| \leq(k-1) / 2} \sum_{|\beta|+2|\gamma|=k+1}|t|^{-\frac{|\beta|+2|\gamma|}{4}}\|f\|_{L^{1}\left(\mathbb{R}^{N},|x||\beta||y||\gamma|\right)}+|t|^{-\frac{k+1}{4}}\left\||y|^{\frac{k+1}{2}} f\right\|_{L^{1}\left(\mathbb{R}^{N}\right)} \\
& \leq t^{-\frac{k+1}{4}}\left\|\left(1+|x|^{k+1}+|y|^{\frac{k+1}{2}}\right) f\right\|_{L^{1}\left(\mathbb{R}^{N}\right)} .
\end{aligned}
$$

The proof of Theorem 1.1 is now complete.

Remark 5. Remark that all the terms in the left hand side of the main estimate of Theorem 1.1 have a slower decay than those of the right hand side. Also observe that, with respect to Theorem 3.1, in Theorem 1.1 we have assumed only integrability of $(k+1) / 2$ moments in the variable $y$.

\section{The Heisenberg Group}

First of all we give the definition and describe the main aspects of the Heisenberg group. Given $(z, \theta)$ of $\mathbb{R}^{2 n+1}$ in the form $(z, \theta)=\left(z_{1}, \ldots, z_{n}, z_{n+1}, \ldots, z_{2 n}, \theta\right)$, the composition law on the group is given by

$$
(z, \theta) \circ\left(z^{\prime}, \theta^{\prime}\right)=\left(z+z^{\prime}, \theta+\theta^{\prime}+2 \sum_{j=1}^{n}\left(z_{n+j} z_{j}^{\prime}-z_{j} z_{n+j}^{\prime}\right)\right) .
$$

In this way $\left(\mathbb{R}^{2 n+1}, \circ\right)$ is a Lie group, whose identity element is the origin $(z, \theta)=(0,0)$, the inverse being given by $(z, \theta)^{-1}=(-z,-\theta)$.

Let us now consider the dilations

$$
\delta_{\lambda}: \mathbb{R}^{2 n+1} \rightarrow \mathbb{R}^{2 n+1}, \delta_{\lambda}(z, \theta)=\left(\lambda z, \lambda^{2} \theta\right) .
$$


We have that $\delta_{\lambda}$ is an automorphism on $\left(\mathbb{R}^{2 n+1}, \circ\right)$ for every $\lambda>0$. In this way $\mathbb{H}^{n}=$ $\left(\mathbb{R}^{2 n+1}, \circ, \delta_{\lambda}\right)$ is a homogeneous space. The Lie algebra on $\mathbb{H}^{n}$ is given by the left invariant vector fields

$$
Z_{j}=\partial_{z_{j}}+2 z_{n+j} \partial_{\theta}, Z_{n+j}=\partial_{z_{n+j}}-2 z_{j} \partial_{\theta}, j=1, \ldots, n, \Theta=\partial_{\theta} .
$$

The convolution product of two functions $f$ and $g$ on $\mathbb{H}^{n}$ is defined by

$$
(f * g)(w)=\int_{\mathbb{H}^{n}} f\left(w \circ v^{-1}\right) g(v) d v=\int_{\mathbb{H}^{n}} f(v) g\left(v^{-1} \circ w\right) d w,
$$

where $v^{-1}$ is the inverse of element $v$ with respect to the group operation $\circ$ on $\mathbb{H}^{n}$. Here, $d w$ is the Haar measure on $\mathbb{H}^{n}$ which is exactly the euclidian measure on $\mathbb{R}^{2 n+1}$. It should be emphasized that the convolution on the Heisenberg group is not commutative. However if $P$ is a left invariant vector field on $\mathbb{H}^{n}$ then one sees easily that

$$
P\left(f_{1} * f_{2}\right)=f_{1} * P f_{2}=P f_{1} * f_{2} .
$$

The canonical sub-laplacian on $\mathbb{H}^{n}$ is given by

$$
\Delta_{\mathbb{H}^{n}}=\sum_{j=1}^{2 n} Z_{j}^{2}
$$

In the case of the Heisenberg group $\mathbb{H}^{n}$ the exponential and logarithmic maps (see [1], Ch. 1, p. 48, for the definition of the Exp and Log maps on general homogeneous Lie groups) are given by (see [1], p. 167 and Remark 3.2.4 on p. 163)

$$
\operatorname{Exp}\left(\sum_{j=1}^{2 n} z_{j} Z_{j}+\theta \Theta\right)=(z, \theta), z=\left(z_{1}, \ldots, z_{2 n}\right)
$$

and

$$
\log (z, \theta)=\sum_{j=1}^{2 n} z_{j} Z_{j}+\theta \Theta
$$

We point out that properties (4.28) and (4.29) are not true in general for homogeneous Lie groups as we can see in the following example. Following [1], Section 3.2, p. 158 let us set $\mathbb{R}^{N}=\mathbb{R}^{m} \times \mathbb{R}^{n}$ and denote its points $z=(x, \theta)$ with $x \in \mathbb{R}^{m}$ and $\theta \in \mathbb{R}^{n}$. Given a $m \times m$ matrix $B$ with real entries let

$$
(x, \theta) \circ(\xi, \tau)=\left(x+\xi, \theta+\tau+\frac{1}{2}\langle B x, \xi\rangle\right),
$$

where $\langle\cdot, \cdot\rangle$ stands for the inner product in $\mathbb{R}^{m}$. Again $\delta_{\lambda}: \mathbb{R}^{N} \rightarrow \mathbb{R}^{N}, \delta_{\lambda}(x, \theta)=\left(\lambda x, \lambda^{2} \theta\right)$ is an automorphism of $\left(\mathbb{R}^{N}, \circ\right)$ for any $\lambda>0$. Then $\left(\mathbb{R}^{N}, \circ, \delta_{\lambda}\right)$ is a homogeneous Lie group of step two. In this case the Exp and Log functions are given by (see (3.11), p. 167 in [1])

$$
\operatorname{Exp}((\xi, \tau) \cdot Z)=\left(\xi, \tau+\frac{1}{4}\langle B \xi, \xi\rangle\right)
$$


and

$$
\log (x, \theta)=\left(x, \theta-\frac{1}{4}\langle B \xi, \xi\rangle\right) \cdot Z
$$

where

$$
(\xi, \tau) \cdot Z=\sum_{j=1}^{m} \xi_{j} X_{j}+\sum_{i=1}^{n} \tau_{i} \Theta_{i}
$$

and $X_{1}, \ldots, X_{m}, \Theta_{1}, \ldots, \Theta_{n}$ is the Jacobian basis.

We can see that (4.28) and (4.29) hold if and only if $B$ is a skew-adjoint matrix. In the case of $\mathbb{H}^{n}$ this holds since the matrix $B$ is given by

$$
B=4\left(\begin{array}{cc}
0 & \mathbb{I}_{n} \\
-\mathbb{I}_{n} & 0
\end{array}\right)
$$

In the particular case of skew-symmetric matrices $B$ we believe that the results of this section are still valid.

We now recall some results concerning Taylor's formula with integral remainder on homogenous Carnot groups $(\mathbb{G}, 0)$.

Lemma 4.1 (Lemma 20.3.8, [1]). Let $x, g \in \mathbb{G}$ and $u \in C^{m}(\mathbb{G}, \mathbb{R})$. Then we have

$$
u(x \circ h)=\sum_{k=0}^{m} \frac{1}{k !}\left((\log h)^{k} u\right)(x)+\frac{1}{m !} \int_{0}^{1}(1-s)^{m}\left((\log h)^{m+1} u\right)(x \circ \operatorname{Exp}(s \log h)) d s .
$$

Let us now apply this Lemma to the case of the Heisenberg group $\mathbb{H}^{n}$. Using the structure on $\mathbb{H}^{n}$ we obtain that for any $h \in \mathbb{H}^{n}$ and $s \in \mathbb{R}$ the following holds

$$
\operatorname{Exp}(s \log h)=s h .
$$

Thus Lemma 4.1 (see Corollary 20.3. 9 in [1], p. 755) gives us the following:

Lemma 4.2. Let $x, h \in \mathbb{H}^{n}$ and $u \in C^{m+1}\left(\mathbb{H}^{n}, \mathbb{R}\right)$. Then we have

$$
\begin{aligned}
u(x \circ h)= & \sum_{k=0}^{m} \sum_{i_{1}, \ldots, i_{k}=1}^{2 n+1} \frac{h_{i_{1}} \ldots h_{i_{k}}}{k !}\left(Z_{i_{1}} \ldots Z_{i_{k}} u\right)(x) \\
& +\sum_{i_{1}, \ldots, i_{m+1}=1}^{2 n+1} \frac{h_{i_{1}} \ldots h_{i_{m+1}}}{(m+1) !} \int_{0}^{1}(1-s)^{m}\left(Z_{i_{1}} \ldots Z_{i_{m+1}} u\right)(x \circ s h) d s,
\end{aligned}
$$

where $h_{2 n+1}=\theta$ and $Z_{2 n+1}=\Theta$.

We now follow the same ideas as in the case of the anisotropic heat equation making partial decompositions in some of the involved variables. Here, in the context of the Heisenbergh group, variables $z=\left(z_{1}, \ldots, z_{2 n}\right)$ and $z_{2 n+1}=\theta$ will be treated separately. For simplicity we treat here only the case $m=1$. 
Lemma 4.3. For any $\varphi \in \mathcal{D}\left(\mathbb{R}^{2 n+1}\right)$ the following identity holds:

$$
\begin{aligned}
\varphi(z, \theta)= & \varphi(0,0)+\sum_{j=1}^{2 n} z_{j}\left(Z_{j} \varphi\right)(0,0) \\
& +\int_{0}^{1}(1-s) \sum_{j, k=1}^{2 n} z_{j} z_{k}\left(Z_{j} Z_{k} \varphi\right)(s z, 0) d s+\int_{0}^{1} \theta(\Theta \varphi)((z, s \theta)) d s .
\end{aligned}
$$

Remark 6. We emphasize the difference with formula (20.63) in [1], p. 761:

$$
\varphi(z, \theta)=\varphi(0,0)+\sum_{j=1}^{2 n} z_{j}\left(Z_{j} \varphi\right)(0,0)+\theta(\Theta \varphi)(0,0)+\sum_{j, k=1}^{2 n} \int_{0}^{1}(1-s) z_{j} z_{k}\left(Z_{j} Z_{k} \varphi\right)(s z, s \theta) d s .
$$

Proof. We apply Lemma 4.2 to $x=(z, 0)$ and $h=(0, \theta)$, elements from $\mathbb{R}^{2 n} \times \mathbb{R}$. Thus

$$
\varphi(z, \theta)=\varphi(z, 0)+\int_{0}^{1}(\theta \Theta \varphi)((z, 0) \circ(0, s \theta)) d s=\varphi(z, 0)+\int_{0}^{1} \theta(\Theta \varphi)((z, s \theta)) d s .
$$

We now apply Lemma 4.2 with $x=(0,0)$ and $h=(z, 0)$ :

$$
\varphi(z, 0)=\varphi(0,0)+\sum_{j=1}^{2 n} z_{j}\left(Z_{j} \varphi\right)(0,0)+\int_{0}^{1}(1-s) \sum_{j, k=1}^{2 n} z_{j} z_{k}\left(Z_{j} Z_{k} \varphi\right)(s z, 0) d s .
$$

Putting toghether the above identities we obtain that

$$
\begin{aligned}
\varphi(z, t)= & \varphi(0,0)+\sum_{j=1}^{2 n} z_{j}\left(Z_{j} \varphi\right)(0,0)+\int_{0}^{1}(1-s) \sum_{j, k=1}^{2 n} z_{j} z_{k}\left(Z_{j} Z_{k} \varphi\right)(s z, 0) d s \\
& +\int_{0}^{1} \theta(\Theta \varphi)((z, s \theta)) d s
\end{aligned}
$$

and the proof is now finished.

We now apply the Taylor like identity in Lemma 4.3 to obtain a decomposition of a function $f$ into Dirac's delta basis adapted to the Lie algebra on $\mathbb{H}^{n}$.

Theorem 4.1. For any $f \in L^{1}\left(\mathbb{R}^{2 n+1}, 1+|z|^{2}+|\theta|\right)$ the following decomposition holds:

$$
f=\left(\int_{\mathbb{R}^{2 n+1}} f\right) \delta_{0}+\sum_{j=1}^{2 n}\left(\int_{\mathbb{R}^{2 n+1}} z_{j} f(z, \theta) d z d \theta\right) Z_{j} \delta_{0}+(\Theta F)+\sum_{j, k=1}^{2 n} Z_{j} Z_{k}\left(F_{j k} \delta_{0}(\theta)\right),
$$

where

$$
F(z, \theta)=-\int_{0}^{1} \frac{\theta}{s} f\left(z, \frac{\theta}{s}\right) d s
$$

and

$$
F_{j k}(z)=\int_{\mathbb{R}} \int_{0}^{1}(1-s) \frac{z_{j}}{s} \frac{z_{k}}{s} f\left(\frac{z}{s}, \frac{\theta}{s}\right) \frac{d s d \theta}{s^{2 n}} .
$$


Moreover,

and

$$
\|F\|_{L^{1}\left(\mathbb{R}^{2 n+1}\right)} \leq\|\theta f\|_{L^{1}\left(\mathbb{R}^{2 n+1}\right)}
$$

$$
\left\|F_{j k}\right\|_{L^{1}\left(\mathbb{R}^{2 n+1}\right)} \leq\left\|z_{j} z_{k} f\right\|_{L^{1}\left(\mathbb{R}^{2 n+1}\right)} .
$$

Proof. First of all it is sufficient to prove the above theorem for smooth $f$ and then extend the result for any $f \in L^{1}\left(\mathbb{R}^{2 n+1},|z|^{2}+|\theta|\right)$. Let us choose $f$ and $\varphi$ two smooth functions. Applying Taylor's expansion obtained in Lemma 4.3 we get the following:

$$
\begin{aligned}
\langle f, \varphi\rangle_{\mathcal{D}^{\prime}, \mathcal{D}}= & \int_{\mathbb{R}^{2 n+1}} f(z, \theta)\left(\varphi(0,0)+\sum_{j=1}^{2 n} z_{j}\left(Z_{j} \varphi\right)(0,0)\right) d z d \theta \\
& +\int_{\mathbb{R}^{2 n+1}} f(z, \theta) \int_{0}^{1}(1-s) \sum_{j, k=1}^{2 n} z_{j} z_{k}\left(Z_{j} Z_{k} \varphi\right)(s z, 0) d s d z d \theta \\
& +\int_{\mathbb{R}^{2 n+1}} f(z, \theta) \int_{0}^{1} \theta(\Theta \varphi)(z, s \theta) d s d z d \theta \\
= & <\delta_{(0,0)}, \varphi>\int_{\mathbb{R}^{2 n+1}} f(z, \theta) d z d \theta-\sum_{j=1}^{2 n}<Z_{j} \delta_{(0,0)}, \varphi>\int_{\mathbb{R}^{2 n+1}} f(z, \theta) z_{j} d z d \theta \\
& +\sum_{j, k=1}^{2 n} \int_{\mathbb{R}^{2 n+1}}\left(Z_{j} Z_{k} \varphi\right)(z, 0) \int_{0}^{1}(1-s) f\left(\frac{z}{s}, \theta\right) \frac{z_{j}}{s} \frac{z_{k}}{s} \frac{d s}{s^{2 n}} d z d \theta \\
& +\int_{\mathbb{R}^{2 n+1}}(\Theta \varphi)(z, \theta) \int_{0}^{1} \frac{\theta}{s} f\left(z, \frac{\theta}{s}\right) \frac{d s}{s} d z d \theta .
\end{aligned}
$$

Observe now that

$$
\begin{aligned}
\int_{\mathbb{R}^{2 n+1}} & \left(Z_{j} Z_{k} \varphi\right)(z, 0) \int_{0}^{1}(1-s) f\left(\frac{z}{s}, \theta\right) \frac{z_{j}}{s} \frac{z_{k}}{s} \frac{d s}{s^{2 n}} d z d \theta \\
= & \int_{\mathbb{R}^{2 n}}\left(Z_{j} Z_{k} \varphi\right)(z, 0)\left[\int_{\mathbb{R}} \int_{0}^{1}(1-s) f\left(\frac{z}{s}, \theta\right) \frac{z_{j}}{s} \frac{z_{k}}{s} \frac{d s d \theta}{s^{2 n}}\right] d z \\
= & \int_{\mathbb{R}^{2 n}}\left(Z_{j} Z_{k} \varphi\right)(z, 0) F_{j k}(z) d z=\left\langle F_{j k} \delta_{0}(\theta), Z_{j} Z_{k} \varphi\right\rangle_{\mathcal{D}^{\prime}, \mathcal{D}} \\
= & \left\langle Z_{j} Z_{k}\left(F_{j k} \delta_{0}(\theta)\right), \varphi\right\rangle_{\mathcal{D}^{\prime}, \mathcal{D}}
\end{aligned}
$$

where

Denoting

$$
F_{j k}(z)=\int_{\mathbb{R}} \int_{0}^{1}(1-s) f\left(\frac{z}{s}, \theta\right) \frac{z_{j}}{s} \frac{z_{k}}{s} \frac{d s d \theta}{s^{2 n}} .
$$

$$
F(z, \theta)=-\int_{\mathbb{R}^{2 n+1}} \frac{\theta}{s} f\left(z, \frac{\theta}{s}\right) d s
$$

we obtain the desired decomposition. The estimates of the $L^{1}$-norms of $F$ and $F_{j k}$ immediately follow. 
We now apply the decomposition obtained in Theorem 4.1 to obtain the asymptotic behaviour of the solutions of the heat equation on the Heisenbergh group $\mathbb{H}^{n}$.

Proof of Theorem 1.3. Using decomposition (4.31) we have that

$$
\begin{aligned}
\| u(z, \theta, t)- & \left(\int_{\mathbb{R}^{2 n+1}} f\right) H_{t}+\sum_{j=1}^{2 n}\left(\int_{\mathbb{R}^{2 n+1}} z_{j} f(z, \theta)\right) Z_{j} H_{t} \|_{L^{1}\left(\mathbb{R}^{2 n+1}\right)} \\
& \leq\left\|\Theta F * H_{t}\right\|_{L^{1}\left(\mathbb{R}^{2 n+1}\right)}+\sum_{j, k=1}^{2 n}\left\|Z_{j} Z_{k}\left(F_{j k} \delta_{0}(\theta)\right) * H_{t}\right\|_{L^{1}\left(\mathbb{R}^{2 n+1}\right)} \\
& =\left\|F * \Theta H_{t}\right\|_{L^{1}\left(\mathbb{R}^{2 n+1}\right)}+\sum_{j, k=1}^{2 n}\left\|\left(F_{j k} \delta_{0}(\theta)\right) *\left(Z_{j} Z_{k} H_{t}\right)\right\|_{L^{1}\left(\mathbb{R}^{2 n+1}\right)} \\
& \leq\|F\|_{L^{1}\left(\mathbb{R}^{2 n+1}\right)}\left\|\Theta H_{t}\right\|_{L^{1}\left(\mathbb{R}^{2 n+1}\right)}+\sum_{j, k=1}^{2 n}\left\|F_{j k}\right\|_{L^{1}\left(\mathbb{R}^{2 n}\right)}\left\|Z_{j} Z_{k} H_{t}\right\|_{L^{1}\left(\mathbb{R}^{2 n+1}\right)} \\
& \leq\|\theta f\|_{L^{1}\left(\mathbb{R}^{2 n+1}\right)}+\sum_{j, k=1}^{2 n}\left\|z_{j} z_{k} f\right\|_{L^{1}\left(\mathbb{R}^{2 n+1}\right)} \leq\left\|\left(|\theta|+|z|^{2}\right) f\right\|_{L^{1}\left(\mathbb{R}^{2 n+1}\right)} .
\end{aligned}
$$

The proof of the decay rate property is now finished.

\section{Acknowledgements.}

The first author was partially supported by Grant PN-II-ID-PCE-2011-3-0075 of the Romanian National Authority for Scientific Research, CNCS - UEFISCDI, MTM201129306-C02-00, MICINN, Spain and ERC Advanced Grant FP7-246775 NUMERIWAVES.

The second author was partially supported by Grant MTM2011-29306-C02-00, MICINN, Spain, ERC Advanced Grant FP7-246775 NUMERIWAVES, ESF Research Networking Programme OPTPDE and Grant PI2010-04 of the Basque Government.

\section{REFERENCES}

[1] A. Bonfiglioli, E. Lanconelli, and F. Uguzzoni. Stratified Lie groups and potential theory for their sub-Laplacians. Springer Monographs in Mathematics. Springer, Berlin, 2007.

[2] A. Carpio. Long-time Behaviour for Solutions of the Vlasov-Poisson-Fokker-Planck Equation. Mathematical Methods in the Applied Sciences Math. Meth. Appl. Sci., 21, 9851014 (1998).

[3] J. Duoandikoetxea and E. Zuazua. Moments, masses de Dirac et décomposition de fonctions. $C$. $R$. Acad. Sci. Paris Sér. I Math., 315(6):693-698, 1992.

[4] B. Gaveau. Principe de moindre action, propagation de la chaleur et estimées sous elliptiques sur certains groupes nilpotents. Acta Math., 139(1-2):95-153, 1977.

[5] J. Ortega and E. Zuazua. Large time behavior in $R^{N}$ for linear parabolic equations with periodic coefficients. Asymptotic Analysis, 22 (1) (2000), 51-85.

[6] F. Rossi. Large time behavior for the heat equation on Carnot groups, preprint, 2012. 
L. I. IGNAT

Institute of Mathematics "Simion Stoilow" of the Romanian Academy, 21 Calea Grivitei Street, 010702 Bucharest, Romania

AND

BCAM - Basque Center for Applied Mathematics,

Alameda de Mazarredo, 14 E-48009 Bilbao, Basque Country - Spain.

E-mail address: liviu.ignat@gmail.com

Web page: http://www.imar.ro/ lignat

E. ZUAZUA

BCAM - Basque Center for Applied Mathematics,

Alameda de Mazarredo, 14 E-48009 Bilbao, Basque Country - Spain AND

Ikerbasque, Basque Foundation for Science,

Alameda Urquijo 36-5, Plaza Bizkaia, 48011, Bilbao, Basque Country, Spain

E-mail address: zuazua@bcamath.org

Web page: http://www.bcamath.org/zuazua/ 